\title{
Pharmacokinetics of Torsemide in Patients with Decompensated and Compensated Congestive Heart Failure
}

\author{
Barry E. Bleske, PharmD, FCCP, Lynda S. Welage, PharmD, William G. Kramer, PhD, \\ and John M. Nicklas, $M D$
}

Plasma pharmacokinetics of oral furosemide have been shown to be influenced by degree of decompensation in patients with congestive heart failure (CHF). This open-label, sequential comparison trial was conducted to determine whether CHF decompensation also alters the pharmacokinetics and pharmacodynamics of torsemide. Twelve patients with CHF, defined by either hemodynamic parameters or clinical signs and symptoms, were enrolled. On admission for treatment of their CHF, the patients were given $100 \mathrm{mg}$ oral torsemide (phase A). A second dose of oral torsemide $100 \mathrm{mg}$ was administered after hemodynamic parameters and clinical signs and symptoms of decompensated CHF resolved (phase B). Plasma and urine samples were collected over a 24-hour period for determination of torsemide con- centrations and urine sodium. Hemodynamic measurements and physical signs and symptoms also were evaluated. During phase $A$, patients had significantly greater urine output and fractional sodium excretion compared with phase $B$. A significant increase in the area under the plasma concentration-time curve (AUC) was observed during phase $B$ compared with phase $A$. However, no significant differences in maximal excretion rate of torsemide were noted between phase $A$ and phase $B$. Heart failure status slightly affects the plasma pharmacokinetics of torsemide; however, this does not significantly alter the maximal urinary excretion rate of torsemide.

Journal of Clinical Pharmacology, 1998;38:708-714 (1)1998 The American College of Clinical Pharmacology
$\mathbf{E}$ dema associated with congestive heart failure (CHF) is difficult to manage because patients often have altered response to orally administered diuretics. These changes may be due in part to alterations in the pharmacokinetics of loop diuretics in decompensated and compensated heart failure. A previous study has demonstrated that both the rate of absorption and the pharmacodynamic effect of furosemide differs significantly between patients with compensated and decompensated CHF. ${ }^{1}$ Alterations in drug absorption may be the result of changes in gastrointestinal physiology (i.e., delayed gastric emptying, edema, and decreased gastrointestinal motility) that occur in CHF. The change in

From the College of Pharmacy, University of Michigan, and the University of Michigan Health System, Ann Arbor, Michigan (Drs. Bleske and Welage); Clinical Research, Boehringer Mannheim Corporation, Gaithersburg, Maryland (Dr. Kramer); and the Department of Internal Medicine, University of Michigan, Ann Arbor, Michigan (Dr. Nicklas). Dr. Kramer is currently with Quintiles Strategic Regulatory Division, Rockville, Maryland. Supported in part by a grant from Boehringer Mannheim Pharmaceuticals. Submitted for publication February 19, 1998; accepted in revised form April 27, 1998. Address for reprints: Barry E. Bleske, PharmD, University of Michigan, College of Pharmacy, Ann Arbor, MI 48109-1065. diuretic efficacy may relate to changes in urinary excretion of drug, intravascular volume, and diet. Regardless of the mechanism, the variation in these parameters between patients with compensated and those with decompensated disease makes effective treatment of edema in CHF challenging.

Torsemide is a loop diuretic of the pyridine sulfonylurea class approved for the treatment of edema in patients with CHF. Recent data suggest that the bioavailability of torsemide in a variety of edematous disease conditions, including chronic renal insufficiency, cirrhosis, and CHF, is equal to or greater than that observed in healthy volunteers. ${ }^{2-4}$ In addition, there is less variability in the bioavailability of torsemide than in that of furosemide in patients with CHF. ${ }^{4}$ These data suggest that torsemide achieves consistent concentrations of drug in the body across a variety of edematous disease conditions and may theoretically provide consistent drug concentrations in patients with both compensated and decompensated CHF.

We performed this study to determine if the pharmacokinetics and pharmacodynamics of torsemide are influenced by a patient's heart failure status (decompensated versus compensated). The results from 
this study will help determine if torsemide offers any potential advantage for the treatment of CHF.

\section{PATIENTS AND METHODS}

A total of 12 patients completed an open-label sequential trial evaluating the pharmacokinetic and pharmacodynamic properties of torsemide in decompensated (phase A) and compensated CHF (phase B). All patients had been hospitalized for treatment of $\mathrm{CHF}$ at the University of Michigan Health System in Ann Arbor. After approval from the hospital Institutional Review Board and before study entry, informed consent was obtained from each patient. Inclusion criteria for the study included a confirmed diagnosis of chronic CHF $(\geq 3$ months), a New York Heart Association Classification of III or IV, systolic dysfunction defined as a left ventricular ejection fraction $\leq 40 \%$ measured within the past year, and an etiology of CHF secondary to ischemic, idiopathic, or noncritical valvular disease.

In addition, study patients had to have evidence of congestion and decompensation defined by physical and/or hemodynamic abnormalities as follows: 1) Physical evidence of congestion, including pitting edema $\geq 2 \mathrm{~mm}$ in the lower extremities extending from the ankles to mid-calf and rales on pulmonary examination; and 2) hemodynamic evidence of congestion, including pulmonary capillary wedge pressure $\geq 25 \mathrm{mmHg}$ and right atrial pressure $\geq 10$ $\mathrm{mmHg}$.

Hemodynamic measurements were performed by placement of a pulmonary artery catheter whose placement was verified by waveform evaluation. Exclusion criteria included serum creatinine $\geq 3$ $\mathrm{mg} / \mathrm{dL}$, serum bilirubin $\geq 2 \mathrm{mg} / \mathrm{dL}$, requirement for a nonsteroidal antiinflammatory agent (with the exception of low dose aspirin, up to $325 \mathrm{mg} /$ day) or probenecid, and CHF secondary to nondilated cardiomyopathy or hemodynamically significant stenotic valvular disease.

All participants were inpatients admitted to the hospital for the treatment of CHF. On admission, baseline blood and urine samples were obtained, including predose samples for measurement of plasma and urine torsemide concentrations. After the baseline samples were obtained, phase A of the study was initiated with oral administration of torsemide $100 \mathrm{mg}$ with $120 \mathrm{~mL}$ of water. Plasma samples were collected from a forearm venous catheter into heparinized vacutainer tubes (Becton Dickinson, Rutherford, NJ) before and $0.25,0.5,0.75,1.0$, $1.25,1.5,1.75,2.0,2.25,2.5,3.0,4.0,6.0,8.0,12.0$, and 24.0 hours after drug administration. Urine samples were collected and analyzed for torsemide, sodium, and creatinine concentrations before and for the intervals from 0 to 1,1 to 2,2 to 3,3 to 4,4 to 6 ,
6 to 8,8 to 12 , and 12 to 24 hours after administration. After the last sample was obtained, further diuresis was achieved with either bumetanide or furosemide administered orally or intravenously, as indicated based on the patient's clinical status.

For the second phase of the study (phase B), torsemide $100 \mathrm{mg}$ was administered orally with 120 $\mathrm{mL}$ of water after the resolution of the signs of congestion, as evidenced by the following: 1) a decrease in body weight $\geq 5$ pounds; and 2) resolution of the physical findings of congestion or a decrease in hemodynamic abnormalities, including resolution of the physical findings of congestion including peripheral edema and rales on pulmonary examination and a decrease in the hemodynamic abnormalities of congestion defined as a decrease of $\geq 10 \mathrm{mmHg}$ in pulmonary capillary wedge pressure or to an absolute pulmonary capillary wedge pressure $\leq 18$ $\mathrm{mmHg}$ and a decrease in right atrial pressure to an absolute pressure $\leq 8 \mathrm{mmHg}$.

For the second phase of the study, time of food intake and concomitant drug administration were reasonably matched to those of the first phase. The time of torsemide administration was also matched (within a 4-hour time period) to the first phase. Plasma and urine measurements were obtained at the same time points as for the first phase. In patients who showed a minimal response to administration of torsemide during the first phase, the study was terminated no sooner than the 6-hour collection time point. In these cases, data collection time points for the second phase were matched to the first phase.

\section{Drug Analysis}

Plasma and urine concentrations of torsemide were measured by a high-performance liquid chromatography (HPLC) method that has been previously described and validated. ${ }^{5}$ The interday and intraday coefficient of variation over a linear range of $\mathbf{5 0}$ to $2,000 \mathrm{ng} / \mathrm{mL}$ was less than $10 \%$ for determination of both plasma and urine concentrations of torsemide.

\section{Data Analysis}

Pharmacokinetic parameters were determined by noncompartmental methods and inspection of the data where appropriate. Specifically, the area under the plasma concentration-time curve (AUC) to the last measured time point was determined by the linear trapezoidal method with extrapolation to infinity. The elimination half-life $\left(t_{2}^{1}\right)$ was determined by linear regression analysis of the terminal phase of the log concentration-time profile. The maximal plasma concentration $\left(C_{\max }\right)$ and time to $C_{\max }\left(t_{\max }\right)$ were determined by inspection of the available data points. Mean residence time (MRT) was calculated 
Table I Mean Clinical Parameters in Patients with Decompensated (Phase A) and Compensated (Phase B) Congestive Heart Failure

\begin{tabular}{lcc}
\hline \hline Parameter & Phase A & Phase B \\
\hline Weight (kg) & $84.1 \pm 22.2$ & $79.5 \pm 21.0^{\star}$ \\
RAP (mmHg) & $13.1 \pm 3.9$ & $3.9 \pm 2.1^{\star}$ \\
PAS (mmHg) & $64.7 \pm 10.8$ & $32.6 \pm 8.7^{\star}$ \\
PAD (mmHg) & $35.3 \pm 4.5$ & $16.2 \pm 5.0^{\star}$ \\
PCWP (mmHg) & $36.3 \pm 3.8$ & $12.2 \pm 3.8^{\star}$ \\
BPS (mmH) & $110 \pm 14$ & $103 \pm 5$ \\
BPD (mmHg) & $74 \pm 11$ & $69 \pm 6$ \\
HR (beats/min) & $93 \pm 14$ & $84 \pm 12$ \\
Cl (mL/min) & $88 \pm 31$ & $75 \pm 23^{\star}$ \\
\hline
\end{tabular}

${ }^{\star} P<0.05$ versus phase $A$.

RAP, right atrial pressure; PAS, pulmonary artery systolic pressure; PAD, pulmonary capillary diastolic pressure; PCWP, pulmonary capillary wedge pressure; BPS, systolic blood pressure; BPD, diastolic blood pressure; HR, heart rate; $\mathrm{Cl}_{\mathrm{Cr}}$, creatinine clearance.

Values are presented as mean \pm standard deviation.

by dividing area under the first moment curve (determined by the linear trapezoidal method) by the area under the plasma concentration-time curve from zero to infinity. Renal clearance $\left(\mathrm{Cl}_{\mathrm{r}}\right)$ was calculated as the total amount of unchanged drug excreted into the urine (Ae) divided by the AUC to the last measured collection. The urinary excretion rate of torsemide over each collection interval was calculated by dividing the amount of torsemide excreted during the interval by the duration of the period. The maximum excretion rate $\left(E R_{\max }\right)$ was determine by visual inspection of the data. Creatinine clearance and the natriuretic response, as assessed by fractional sodium excretion $\left(\mathrm{Fe}_{\mathrm{na}}\right)$, were determined based on urinary concentrations and standard formulas.

Statistical comparison between the two phases was performed by a paired t-test. $P \leq 0.05$ was considered the critical probability level. The reported data are represented as the mean \pm standard deviation.

\section{RESULTS}

A total of 14 patients were enrolled in the study, with 12 patients completing both phases. Two patients were excluded, one secondary to receiving probenecid and the other secondary to nausea and vomiting after receiving the first dose of torsemide. The urine data for one patient was excluded from analysis based on inadequate urine collection during the study period. Therefore, plasma data from 12 patients were used for analysis, whereas for analysis of the urine data, only 11 of the 12 patients were evaluated. Eight men and four women were included in the study, with a mean age of $53.5 \pm 9$ years and a mean ejection fraction of $23 \pm 7 \%$. Of the patients who completed the study, nine qualified for the study according to hemodynamic measurements and three qualified based on clinical observations.

Mean clinical parameters for phase A and phase B are shown in Table I. Significant differences between phase A and phase B were noted for weight and hemodynamic measurements. No significant differences were noted for heart rate and blood pressure. However, creatinine clearance was significantly diminished during phase B compared with phase A. Of the 12 patients, $8(67 \%)$ had a decrease in creatinine clearance between phase A and phase B (Figure 1). In addition, significant differences were also noted for both serum creatinine and blood urea nitrogen to serum creatinine ratio between phase $A$ and $B(1.1 \pm$ 0.04 versus $1.6 \pm 0.05 \mathrm{mg} / \mathrm{dL}, P<0.03$, and $16.8 \pm$ 1.9 versus $22.1 \pm 2.9, P<0.02$, respectively). A total of 10 of the 12 patients had increases in both parameters from phase A to phase $B$.

Mean pharmacokinetic parameters for plasma concentrations of torsemide are shown in Table II, and the mean concentration-time profiles are displayed in Figure 2. The mean AUC value was significantly higher during the compensated stage (phase B) than

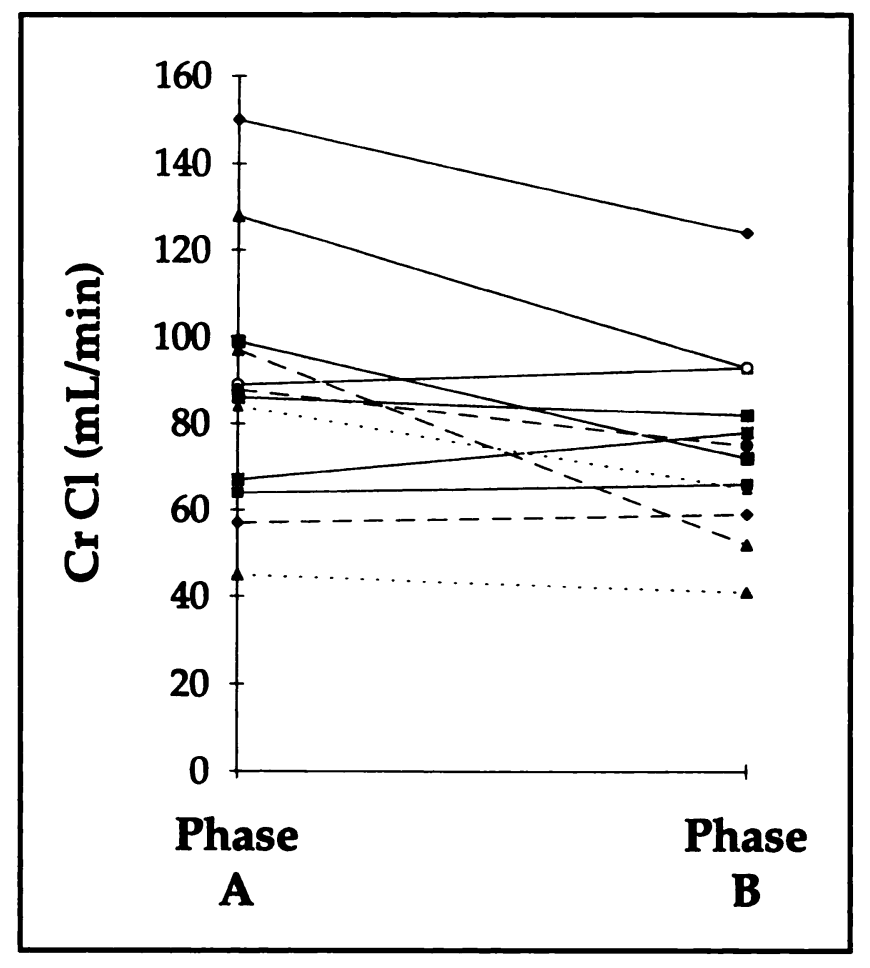

Figure 1. Change in creatinine clearance between Phase $A$ (decompensated) and Phase $B$ (compensated) for individual patients. Each symbol represents an individual patient. The mean is indicated by the closed circles. 
Table II Mean Pharmacokinetic Data for Patients with Decompensated (Phase A) and Compensated (Phase B) Congestive Heart Failure

\begin{tabular}{lcc}
\hline \multicolumn{1}{c}{ Parameter } & Phase A & Phase B \\
\hline $\mathrm{C}_{\max }(\mu \mathrm{g} / \mathrm{mL})$ & $10.1 \pm 3.4$ & $12.7 \pm 4.6$ \\
$\mathrm{t}_{\max }(\mathrm{hrs})$ & $2.7 \pm 3.0$ & $1.5 \pm 0.6$ \\
$\mathrm{AUC}(\mathrm{hr} \cdot \mu \mathrm{g} / \mathrm{mL})$ & $39.9 \pm 13.0$ & $47.2 \pm 18.1^{\star}$ \\
$\mathrm{t}_{1 / 2}(\mathrm{hrs})$ & $4.2 \pm 1.2$ & $4.3 \pm 1.3$ \\
$\mathrm{MRT}^{\prime}(\mathrm{hrs})$ & $5.87 \pm 1.9$ & $5.5 \pm 1.7$ \\
$\mathrm{Cl}_{\mathrm{r}}$ & $7.2 \pm 3.2$ & $4.6 \pm 2.5^{\star}$ \\
$\mathrm{Ae}(\%$ dose $)$ & $16.1 \pm 9.0$ & $12.1 \pm 6.5$ \\
$\mathrm{ER}_{\max }(\mu \mathrm{g} / \mathrm{hr})$ & $3729 \pm 2087$ & $3378 \pm 1556$ \\
\hline
\end{tabular}

${ }^{*} P<0.05$ versus phase $A$.

Values are presented as the mean \pm standard deviation.

$\mathrm{C}_{\max }$, maximal plasma concentration; $t_{\max }$, time to $\mathrm{C}_{\max }$; $A U C$, area under the concetration-time curve; $1_{1 / 2}$, half-life; MRT, mean residence time; $\mathrm{Cl}_{r}$, renal clearance; $\mathrm{Ae}$, unchanged drug excreted into the urine; $\mathrm{ER}_{\max }$, $\max$ imum urinary excretion rate.

in the decompensated stage (phase A). Although mean $C_{\max }$ was higher and mean $t_{\max }$ shorter during phase $B$ compared with phase $A$, these differences were not statistically significant. The longer $t_{\max }$ during phase $A$ is somewhat skewed because one patient had a $t_{\max }$ of 12 hours. Exclusion of this data point shows an average $t_{\max }$ of $1.87 \pm 0.62$ hours (range 0.75-2.5 hours), which is similar to the $t_{\max }$ of $1.54 \pm 0.62$ hours observed during phase B (range 1-3 hours). No statistical differences were noted for the other parameters.

Data regarding urinary excretion of torsemide are shown in Table II. The mean urinary excretion rate time profile of torsemide for each treatment phase is depicted in Figure 3. The maximal urinary excretion rate for torsemide was similar between phase $A$ and phase B. Renal clearance of torsemide was significantly higher during the decompensated phase.

Regarding pharmacodynamic response, the maximal fractional sodium excretion and total sodium was significantly higher for phase $A$ than for phase $B$ $(5.6 \pm 1.9 \%$ versus $3.4 \pm 1.8 \%$ and $73.6 \pm 51 \mathrm{mEq} / \mathrm{hr}$ versus $26.6 \pm 20.2 \mathrm{mEq} / \mathrm{hr}$, respectively; $P<0.03$ ). Overall sodium excretion during the study period was higher during phase $\mathrm{A}$ than during phase $\mathrm{B}$ $(272 \pm 139 \mathrm{mEq}$ versus $89 \pm 57 \mathrm{mEq}$, respectively; $P$ $<0.002$; Figure 4). In conjunction with the effect on sodium excretion, urine volumes during the study period were also significantly greater during phase A than during phase $B(3,338 \pm 1,244 \mathrm{~mL}$ versus $1,903 \pm 688 \mathrm{~mL}$, respectively; $P<0.0007)$.

\section{DISCUSSION}

This study demonstrated that the plasma pharmacokinetics and pharmacodynamic effect of orally ad- ministered torsemide were altered by degree of decompensation in patients with CHF. Based on the plasma pharmacokinetics, one would predict that the pharmacodynamic response would be lower during the decompensated state than the compensated state; however, the urinary pharmacokinetic data suggests the opposite. The urinary pharmacokinetic parameters, including renal clearance, were higher during the decompensated phase than during the compensated phase, and this may explain the greater pharmacodynamic response (i.e., high urine output, $\mathrm{Fe}_{\mathrm{Na}}$, etc) that was observed during the decompensated state. Differences in pharmacodynamic responses between the two study phases may also be explained in part by alterations in physiology that occur secondary to changes in intravascular volume.

Although there was a slight increase in $\mathrm{C}_{\max }$ and a slight decrease in $t_{\max }$ during the compensated phase, no statistically significant differences were detected between the two study phases. Specifically, 10 of the 12 patients had an increase in $C_{\max }$ and 9 of 12 patients had a decrease in $t_{\max }$ during the compensated phase. The lack of statistical significance between the two phases may be the result of a type II error. Overall, the data suggest that the absorption of torsemide maybe slightly slower in some patients during the decompensated state. However, the relatively minor changes in $\mathrm{C}_{\max }$ and $\mathrm{t}_{\max }$ are of questionable clinical importance.

A previous study in which furosemide was administered to patients with decompensated and compensated CHF (based on clinical signs and symptoms) suggests an alteration in absorption. ${ }^{1}$ Specifically, statistically significant differences were observed for $\mathrm{C}_{\max }$ and $\mathrm{t}_{\max }$ values between the two phases. The degree of alteration in $t_{\max }$ and $C_{\max }$ between decompensated and compensated states observed with furosemide was similar to that observed in this study. Overall, the clinical significance of these changes are most likely dependent on the dose used. The precise mechanism responsible for this altered absorption for both drugs is not known, but may relate to changes in gastrointestinal physiology (i.e., delayed gastric emptying, edema, and decreased gastrointestinal motility) that may occur during the decompensated phase of CHF, or to changes in volume of distribution.

There was an increase in AUC during the compensated phase compared with the decompensated phase, with an AUC ratio of $86.4 \%$ (decompensated to compensated). The increase in AUC after compensation may be related to changes in absorption $\left(C_{\max }\right)$, renal clearance, total clearance, or even volume of distribution (secondary to fluid changes). Although we can not definitively identify which of the above factors are responsible for the change in AUC, the change in renal clearance observed in this 


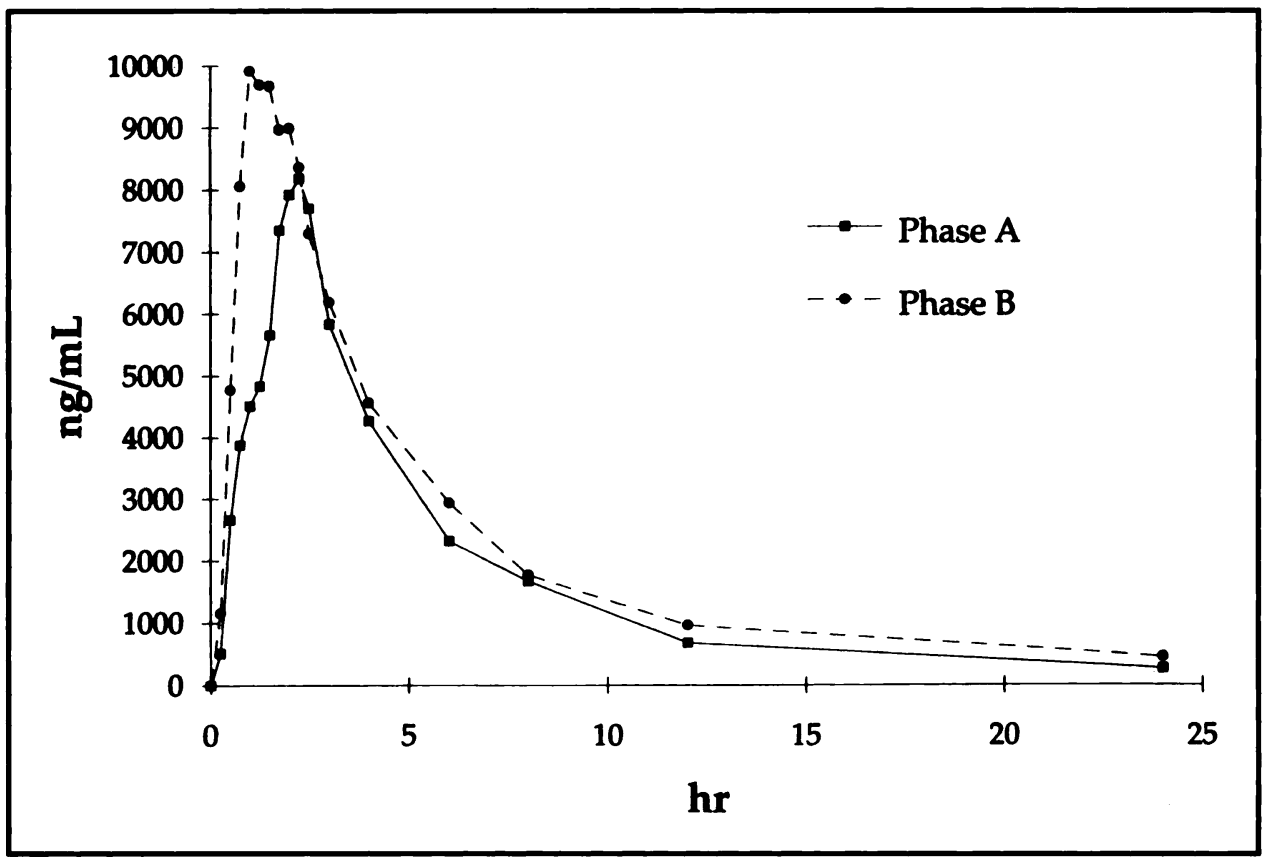

Figure 2. Mean torsemide plasma concentration-time curve in patients with decompensated (Phase A) and compensated (Phase B) heart failure. study is unlikely to be the sole contributing factor. Renal clearance accounts for no more than $25 \%$ of total torsemide clearance in subjects with normal kidney function, ${ }^{2}$ and thus the change in renal clearance observed in this study would not predict the change seen in AUC. A decrease in total clearance could also lead to an increased AUC. To definitely determine if the extent of absorption is affected or if there is a change in clearance, administration of intravenous torsemide is required.

For torsemide to exert its effect, drug must reach the urine, because the site of action for a loop diuretic is the luminal side of the tubule. ${ }^{6}$ Therefore, alterations in the pharmacokinetic parameters of

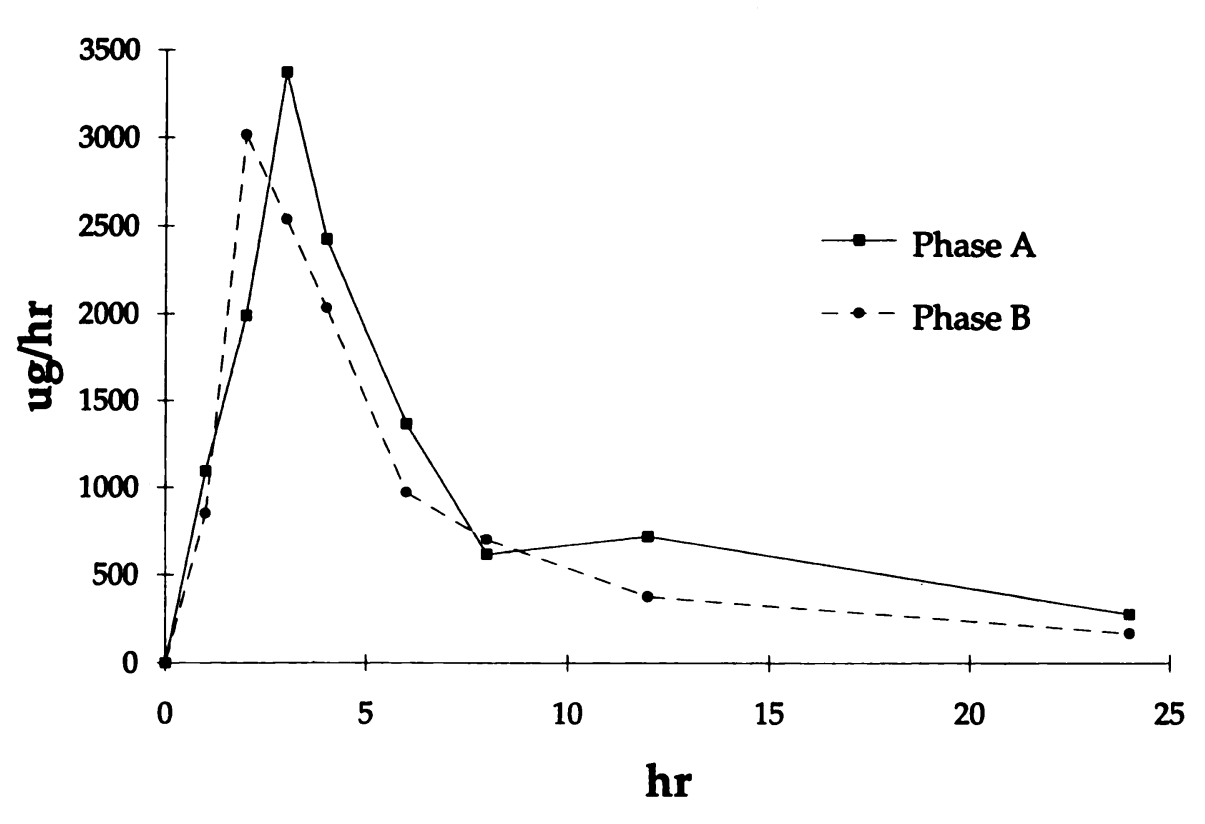

Figure 3. Mean urinary concentration-time curve of torsemide in patients with decompensated (Phase A) and compensated (Phase B) heart failure. 
Figure 4. Mean urinary sodium excretion versus time in patients with decompensated (Phase A) and compensated (Phase B) heart failure.

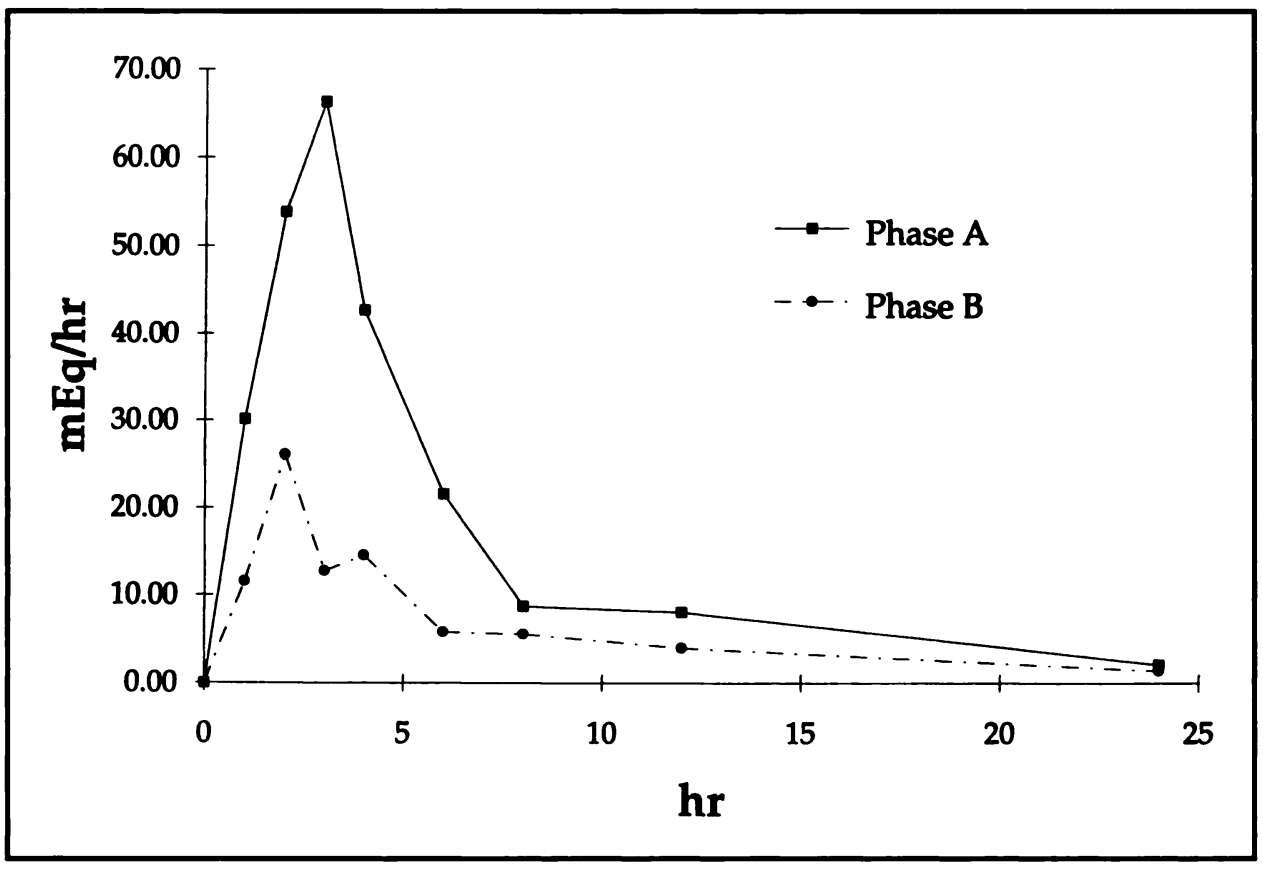

torsemide in plasma observed between the two phases may not be clinically important, because the pharmacokinetic parameters for urinary concentrations of torsemide were similar between the two phases. Specifically, the total amount of drug excreted in the urine, the urinary excretion rate time profile, and the maximal excretion rate of torsemide were similar between the phases (Figure 3). What influence the change in creatinine clearance has on these results is not certain.

The correlation between renal clearance of drug and creatinine clearance was statistically significant but was not equal to one $\left(r^{2}=0.29 ; P<0.05\right)$. In addition, the mean percent change in creatinine clearance between the two phases was less than $20 \%$. These findings suggest that the change in creatinine clearance between the two phases probably had an effect on our results, including on the renal clearance of torsemide, but that these effects were minimal. In other words, urinary concentrations of torsemide would have been similar between the two phases even if there was no statistical difference in creatinine clearance. However, despite our conjectures, we cannot rule out that our findings are directly related to the change in creatinine clearance observed between the two phases. Theoretically, urinary concentrations of torsemide may have been greater in phase B if it were not for the observed change in creatinine clearance.

The diuretic effect of torsemide was greater during the decompensated phase than during the compensated phase of this study. Specifically, urine vol- umes, total sodium excretion, and maximal sodium excretion rate were greater during phase A than during phase B. Fractional excretion of sodium was evaluated to account for differences in creatinine clearance between the two phases. The reason for the diminished response seen in phase $B$ is not clear, but probably relates to what has been described in previous trials as a "braking" effect. ${ }^{1,7-9}$ The mechanism for this temporary condition is not certain, but may relate to physiologic responses that occur secondary to changes in intravascular volume. The clinical significance of this "braking" effect is important, especially with regard to prescribing adequate doses of diuretics on discharge from the hospital. Clinicians need to be cognizant of the diminished diuretic response before discharge and provide appropriate conversion from intravenous to oral formulations with an adequate time period for observation.

A potential limitation of this study is whether or not there was a significant difference in fluid status between the two phases. As shown in Table I, significant differences were observed in intravascular pressures and weight, which suggests that significant differences were obtained. Another consideration is whether the time between the two phases was sufficient to allow for changes in gastrointestinal physiology to occur. The interval between the two phases (5.3 \pm 3.2 days) was similar to that in the previously mentioned furosemide study, in which alterations in pharmacokinetic parameters were observed.

Overall, the results from this study suggest that the plasma pharmacokinetics and effect of torsemide 
may depend on a patient's heart failure status. However, the difference in the plasma pharmacokinetic parameters of torsemide between patients with decompensated and compensated heart failure do not appear to significantly effect the maximal urinary excretion rate of torsemide. This suggests that any change in the clinical response to torsemide between the decompensated and compensated stages of $\mathrm{CHF}$ at the dose studied is probably not related to pharmacokinetic alterations.

\section{REFERENCES}

1. Vasko MR, Cartwright DB, Knochel JP, Nixon JV, Brater DC: Furosemide absorption altered in decompensated congestive heart failure. Ann Intern Med 1985;102:314-318.

2. Gehr TWB, Rudy DW, Matzke GR, Kramer WG, Sica DA, Brater DC: The pharmacokinetics of intravenous and oral torsemide in patients with chronic renal insufficiency. Clin Pharmacol Ther 1994;56:31-38.

3. Schwartz S, Brater DC, Pound D, Green PK, Kramer WG, Rudy D: Bioavailability, pharmacokinetics, and pharmacodynamics of torsemide in patients with cirrhosis. Clin Pharmacol Ther 1993; 54:90-97.

4. Vargo DL, Kramer WG, Black PK, Smith WB, Serpas T, Brater DC: Bioavailability, pharmacokinetics, and pharmacodynamics of torsemide in patients with congestive heart failure. Clin Pharmacol Ther 1995;57:601-609.

5. March C, Farthing D, Wells B, Besenfelder E, Karnes HT: Solid phase extraction and liquid chromatography of torsemide and metabolites from plasma and urine. J Pharm Sci 1990;79:453-457.

6. Cavalier R, Pellegrin P, Lesne J, van Ypersele de Strihou CH: Site of action of torsemide in man. Eur J Clin Pharmacol 1986; 31(suppl):15-19.

7. Hammarlund MM, Odlind B, Paalzow KW: Acute tolerance to furosemide diuresis in humans: pharmacokinetic-pharmacodynamic modeling. J Pharmacol Exp Ther 1985;233:447-453.

8. Li T, Lee MG, Chiou WL: Effects of the rate and composition of fluid replacement on the pharmacokinetics and pharmacodynamics of intravenous furosemide. J Pharmacokinet Biopharm 1986; 14:495-509.

9. Wilcox CS, Mitch WE, Kelly RA, Skorecki K, Meyer TW, Friedman PA, Souney PF: Response of the kidney to furosemide. I. Effects of salt intake and renal compensation. J Lab Clin Med 1983;102:450-458. 